\title{
An Efficient Buffer Overflow Control Scheme to Mitigate Packet Loss in Manets
}

\author{
B Purushotham, Ch D V Subba Rao
}

\begin{abstract}
MANET (Mobile Ad hoc Network) is self-configuring, self-organizing, infrastructure-less network of mobile devices connected without wires. Because of the dynamic changes in the network topology, there is no centralized control in ad hoc networks. So, mobile nodes can communicate with each other via intermediate nodes. The buffer size of intermediate nodes plays a significant role to hold packets in the buffer before dropping the newly arrived packets. In MANET, buffer overflow (congestion) occurs in any intermediary nodes when data packets pass through source to destination and incurs packet loss, which causes the performance degradations of a network. Congestion can be reduced by using several Active Queue Management [AQM] techniques. Queue Management Node or "QMN" is an active queue management strategy to control packet drops by assigning space of a buffer node to all its neighbouring nodes dynamically depending upon the total number of packets received from its neighbours. In this scheme, authors did not take into account the impact of collaboration between neighbors of a central node. They only consider the characteristics of nodes, while ignoring the message properties in MANET. And also a new node has to be waiting until it will get enough equal space from the QMN along with other neighbor nodes. During this waiting period, high packet loss can be occurred. So, an efficient buffer overflow control scheme is required to make sure every node has adequate storage space to hold the data once the buffer of the node is near to congestion. In this paper, we proposed a scheme that integrates QMN technique with combinational buffer management (CBM) strategy, which includes both the features of messages and nodes, and transfer the duplicated messages to neighbour node for total utility optimization, rather than deleting them. By using this scheme packet loss can be mitigated in MANETs efficiently. Our simulation results here reveal that, the proposed scheme achieves better performance than other existing schemes in terms of packet delivery ratio, throughput, overhead ratio and end to end delay .
\end{abstract}

Keywords: Buffer Overflow, Mobile Ad hoc Networks, Packet Loss, Queue Management

\section{INTRODUCTION}

MANETs are self-configuring networks of independent mobile wireless nodes without additional infrastructure or central administration [1]. These networks brought an

Revised Manuscript Received on February 05, 2020.

* Correspondence Author

B.Purushotham*, Dept. of Computer Science and Engineering, S V University, Tirupati, India. Email:purush_bmp@yahoo.com

Dr. Ch D V Subba Rao, Dept. of Computer Science and Engineering, S V University, Tirupati, India. Email:subbarao_chdv@hotmail.com

(C) The Authors. Published by Blue Eyes Intelligence Engineering and Sciences Publication (BEIESP). This is an open access article under the CC BY-NC-ND license (http://creativecommons.org/licenses/by-nc-nd/4.0/) innovative design of networks and best fit for an environment where either the infrastructure is lost. Communication between the end nodes in MANETs can be done through intermediate nodes. The responsibility of the each intermediate node is to receive and forward packets from source to destination. In this connection, incoming packets are queued in the buffer of nodes for their turn of processing. The size of buffer plays a vital role with respect to number of packets that can be held in a queue before dropping newly arrived packets. Congestion causes packet loss at buffers of intermediate nodes in the network i.e packet loss may occur if the size of the buffer becomes full.

A Communication networks normally contains network of queues. Effective handling of queues is needed to allow Quality of Service (QoS). With the support of congestion avoidance and control mechanisms this task can be achieved. This process is called queue management [2]. Queue management contains algorithms that manage size of packet queues by dropping packets whenever required or suitable. Queue management schemes are of two types:

\section{i. Passive Queue Management (PQM)}

ii. Active Queue Management (AQM)

Packets are simply dropped when buffer gets full in PQM. Even though this approach is less effective and has several disadvantages, the main benefit of using PQM is easy to implement in network with less computational overheads.

Drop Tail is the simplest PQM technique. In this technique, packets can be rejected whenever queue gets full. Therefore, in this scheme, size of buffer is the key factor that reduces the packet drop. However, these results in the familiar problem called 'global synchronization' problem [3]. To avoid this problem, Floyd et al. proposed a new scheme called Random Early Detection (RED) [4]. This lead to encourage of a new discipline namely 'Active Queue Management', the principle of which is to drop / mark packets before a queue overflows so that sources respond to congestion thereby avoiding buffer overflow. AQM controls packet loss and increases resource utilization leading to improvement QoS in contrast to drop tail technique [3]. Algorithms like RED, Adaptive RED (ARED), BLUE etc., are examples for Active Queue Management Techniques. RED (random early detection) is the widely used algorithm to monitor the average queue size and take actions on packet (either drop or mark) depending on statistical probabilities.

On the other hand, the mobile and wireless networks still use Drop Tail policies for packet queue management and the efforts to achieve AQM in those networks are very limited. It is observed when AQM is implemented in the nodes of MANET, 
they still lack in efficient queue management because packets are usually treated from other wireless nodes only on the basis of services and applications. On the other hand, MANET nodes are resource constrained devices which have limited battery life and memory/storage spaces. In such conditions, data sent from sources which transmit packets with less data rates did not acquire a fair share in queues.

Muhammad Aamir and Mustafa A. Zaidi proposed a technique to handle packet queues in MANETs for static and mobile nodes [5]. Efficient queuing in the buffer can be achieved by using a centrally communicating node called QMN. This scheme can allocate buffer space of QMN to all its neighboring nodes dynamically depending on the number of packets received from neighbors and thereby reducing packet drop probabilities. In this scheme, authors did not considered the effect of collaboration between the neighboring nodes. They only consider the characteristics of nodes, while ignoring the message attributes in MANET.

Peiyan Yuan and Hai Yu proposed CBM scheme that combines both the attributes of messages and nodes, and transfer messages to neighboring nodes to enhance the total utility, rather than removing them [6]. Taking into account the above facts, we presented an efficient buffer management scheme by combining the QMN with CBM strategy which considered the characteristics of messages and nodes; in the meantime, this scheme also contains message migration mechanism that effectively removes and transfers messages.

Performance evaluation of proposed scheme is conducted using ns-2 simulator. Simulation results reveals the proposed scheme achieves better results than the other existing schemes with respect four metrics: Packet delivery ratio, throughput, overhead ratio and end to end delay respectively. The following are the contributions of this research work:

- Integrate queue management node scheme (QMN) with CBM strategy to allocate buffer space dynamically and decision will be taken whether to utility value the message and that of the node.

- Transfer messages to neighbouring nodes, instead of removing them whenever the buffer capacity of nodes is filled.

The remainder of the paper is planned as follows. Earlier works done by other researchers are reviewed in section 2 . Proposed scheme is presented in section 3. Simulation results are depicted in section 4 . In section 5 conclusions are presented.

\section{REVIEW ON RELEVANT WORK}

Muhammad Aamir and Mustafa A. Zaidi present a technique to manage packet queues in MANETs. In this technique, authors try to achieve effective queuing in the buffer of a centrally communicating node by allocating buffer space dynamically to its neighboring nodes depending on the number of packets received from neighbors [5]. Simulation study reveals that their proposed scheme is a way to improve the buffer management for packet queues in MANETs. Peiyan Yuan and Hai Yu presented a buffer management scheme by combining the characteristics of messages and nodes, and transfer the duplicated messages to the neighboring nodes to enhance the total utility, instead of removing them [6]. Kulkarni et al. presented a technique accept a newly arrived message or not is basing on the

called PAQMAN in contrast to conventional RED algorithm in Real Time IP networks [2]. Their proposed scheme works on predictive measurement of queue size on the basis of traffic variations. The average queue length is predicted for next specified interval using recursive least square method and it determines the packet drop probability in that interval. Their simulation study in NS-2 depicts that the proposed scheme provides better QoS evaluations as compared to RED.

The same scheme is tested by Kulkarni et al. on MANET environment and simulation study is performed in NS-2 for packet loss and retransmission efficiencies to check if the proposed scheme can work in an energy-efficient manner [7]. The simulation results show that PAQMAN coupled with ECN can reduce packet loss ratio and increase transmission efficiency while introducing negligible overhead.

Lutz et al. focused on the assignment of transmission frames with equal number of transmission slots per frame to a wireless node on a channel shared with other nodes [8]. They proposed a variation in such a way that number of transmission slots ("weights") can be varied in different transmission frames. In this way, throughput may be increased without compromising "fairness" and packet losses due to collision may also be mitigated.

Through Drop Tail technique, Chen and Bensaou proposed a study for high speed networks regarding their survivability in terms of fairness and packet loss problems [9]. The authors states that with TCP flows that comes from multiple congested links cause packet drop probability unfairness and round trip time unfairness problems. On the other hand, AQM schemes reduce the severity of above mentioned unfairness.

Abbasov and Korukoglu improved the existing RED algorithm on networks and the improvement is called Effective RED (ERED) [10]. It has a few variations as compared to RED in the packet drop function which produce better throughput and less packet loss rate as compared to RED and some other well known AQM schemes. It is shown by authors through simulation study in NS-2. S. Dimitriou and V. Tsaoussidis demonstrated how SQM classifies traffic and how it applies different policies to each packet depending on its size, the sizes of packets currently in the queue and the contention levels in the router [11].

Hence, it differentiates time-sensitive traffic and applies different policies of scheduling and packet drop on separate flows to increase the level of application satisfaction. Krifa et al. projected a buffer management scheme supported a distributed algorithm [12]. The aim of this scheme is to cut back the average delivery delay or to extend the average delivery ratio. One more vital parameter to valuation principles of the message is TTL (Time to Live) of the message. The Message lifetime time grows longer if the delivery probability of the message increases. If the TTL value of the packet is zero then the packet can be removed. For example, Scott et al. described the TTL value as a timeout value and delete messages whose lifecycle crosses the TTL to avoid congestion within the buffer of a node [13]

Published By: 
It is important to note that, as the range of message copies will increase the likelihood of the message inbound to the destination node will increase. But, too several copies of messages within the network will be cause buffer overflow. Hence, the way to take away the copy of the message and check that the delivery quantitative relation of the message is become a centralized issue. Kim et al. take away the message that has larger range of copies to attenuate the impact of message drop that incurs high delivery quantitative relation in a Delay Tolerant Networks [14].

Message size is used to determine the significance of the message in the buffer of a node. For instance, there is a prospect for the prevalence congestion is going to be decreased if the larger and unusable messages are removed [15]. Rashid et al. projected a way that drops the message within the buffer of a node if its size is bigger than or capable the coming message in [16].

A comprehensive-integrated buffer management (CIM) scheme presented by Pan et al. considered all data related to message delivery and network resources [17]. This scheme computes the utility value of the package from the subsequent characteristics: the message life time, the message size, and therefore the variety of forwarding hops. Yao et al. computes the utility price of caching knowledge betting on node curiosity and knowledge transfer likelihood by as well as passive and proactive dropping policy, and programming policy [18]. Their projected buffer management technique permits the node to be proactively chosen and receive messages with different nodes.

Our literature review identifies that significant amount effort has been done on buffer management in wireless and wired networks. However, a highly responsive solution is required that can address the packet drop issue of queue management in buffers of MANET nodes.

\section{PROPOSED MODEL}

The proposed model integrates QMN and CBM schemes by considering the characteristics of the message and characteristics of the node in MANETs. In first step, the buffer space of a node is assigned equally to all its neighbouring nodes. Figure 1 shows fixed QMN node in mobile adhoc network.

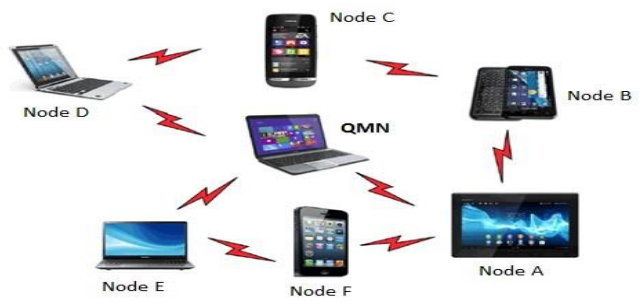

Fig. 1.QMN node in MANET

Whenever the algorithm is triggered on the occurrence of a selected incident, the allotment of buffer area of a node is dynamically adjusted. A fair share in the buffer is available to all neighbors around a MANET node irrespective of packet transmission rates of neighboring sources. Since all neighbors share the buffer space, no node can occupy the buffer in full through aggressively sending packets with higher data rate. A neighbor has its share in the QMN's buffer till it remains a neighbor of QMN.

\section{A. Network model}

Mobile adhoc network can be represented using a dynamic graph $\mathrm{G}=(\mathrm{V}, \mathrm{E})$ where $\mathrm{V}$ denotes set of nodes/vertices, and $\mathrm{E}$ denotes set of links/edges. Every node in the MANET is assigned with a unique identifier. If the nodes are within the communication range they can exchange data. We consider MANET model is operated on Ad hoc On demand Distance Vector (AODV) routing protocol. It is a reactive protocol in which sources get routes to destinations when they demand for the same. Nodes only know their neighbors through routing table entries and keep track of neighbors by exchanging HELLO packets periodically. And also we assume the following properties of MANETs:

- Every node in MANET has limited buffer.

- Node mobility is self-governing and the nodes have diverse contact rates.

- Bandwidth is equal for all the links.

\section{B. Neighbor Node discovery}

In MANETs, the communication is happen through multi-hop transmission. It is necessary to establish the neighbor nodes under such networks. In this module, one hop neighbors of every node is identified dynamically. By using tree topology communication can be done between the nodes with destination as the root. In first stage tree is constructed as follows: First the sender node broadcast a message with a hop counter. Then the nodes that receiving the message will set the message sender as the parent node, increase the hop counter by one, and broadcast it to their neighboring nodes. Data are moved along the edges of the communication tree.

\section{Queue Management Strategy}

Suppose that there is a static node called "QMN" bounded with 4 neighboring nodes from "Node A" to "Node D".

Assume "Node G" is not a neighbor of QMN at first stage. And also consider the size (storage) of QMN node 100 packets and is unfilled at initial phase. QMN assigns equal storage to neighboring nodes in the starting stage. Assume "bs" is buffer space and "nn" is number of neighboring nodes of QMN, then for every neighbor node the assigned buffer space "abs" is abs $=$ bs/ nn.

Initially every neighboring node will get equal buffer space of 25 packets in the QMN. Suppose if any one of the neighboring node of QMN reaches its maximum assignment and the buffer size is available in overall buffer space because of reason of other neighboring nodes are not communicating or sending packets with respect to QMN. There is a chance

for resource underutilization if we confine a node to an assignment of 25 packets of each, but the buffer space is quiet left in the buffer always. Thus provision for extension of each node is limit of 25 packets upto a quota of remaining buffer space.

\section{Message forwarding and migration}

The data in the nodes are handled by the QMN (Queue management node), which handles the neighbor nodes data management and migration process depend on utility function. The utility function of the message can be calculate by the following equation. 


$$
D(S, H, T)=\alpha \times \frac{s_{i}}{S}+\beta \times \frac{h_{i}}{H_{a v}}+\gamma \times \frac{t_{i}}{T T L^{\prime}}
$$

Where $\alpha+\beta+\gamma=1$, the message size $\mathrm{s}_{\mathrm{i}}$, the number of hops $h_{i}$, TTL is threshold of $t_{i}$, the average no of hops is $H_{a v}$, TTL of the message, $S$ is buffer size of the node. The following algorithm shows the procedure to allow collaboration between neighbor nodes and message forwarding and migration.

\section{Algorithm: For each instance of appearance of a new neighboring node}

1.Compute total storage buffer space engaged every node.

2.Enhance the neighboring total ' $\mathrm{nn}$ ' by ' 1 '.

3.Split the available buffer space using ' $n$ '.

4.Allocate the computed storage spaces to all of its neighboring nodes together with new node.

5.If the required buffer space does not exist for the new node, it has to wait until it will get the space.

6.During waiting time of new node, QMN can initiate collaboration between neighbour nodes and do the following:

Let node $A, B \in V$, where $V$ is the total number of nodes. If node B and node A are contact each other \& node B is the sender then forward message to A.

if node $\mathrm{A}$ has enough free space

Compute the utility value of the message of node B by using the equation

$$
D(S, H, T)=\alpha \times \frac{s_{i}}{S}+\beta \times \frac{h_{i}}{H_{a v}}+\gamma \times \frac{t_{i}}{T T L},
$$

\& transfer the message i into node A //utility value calculation of a message

else

Compute the node A utility value by using equation \& match this with message utility value of message using equation

$$
U_{M}=\frac{\sum_{i=1}^{n}-D(S, H, T)}{n},
$$

if the message utility value of node $\mathrm{B}<=$ Node A utility value // node utility value caluculation

then

node A receive message and store it

else

reject the message

end if

if message i utility value > node A utility value

transfer message with smallest utility value of node A to neighboring node \& transfer message from B to node A end if

Fig. 2. depicts combination of two approaches to handle buffer space of nodes in MANETs. Initially the centrally communicating node QMN, equally allocate buffer space to all its neighbors and that space can be dynamically adjusted based on the need of buffer space required by the neighboring nodes. If any new node enters the transmission range of central node, it can be assigned equal buffer space along with other neighbor nodes if enough space is available with QMN. Otherwise, the new node can be entered into waiting state until it will get sufficient space. During this period, packet drop can be occurred at neighboring nodes. The central node has to processing the messages in the buffers of neighbor nodes and incurs packet drop. Meanwhile, QMN can initiate collaboration between neighbor nodes to overcome the packet drop. This collaboration ensures message forwarding and migration efficiently.

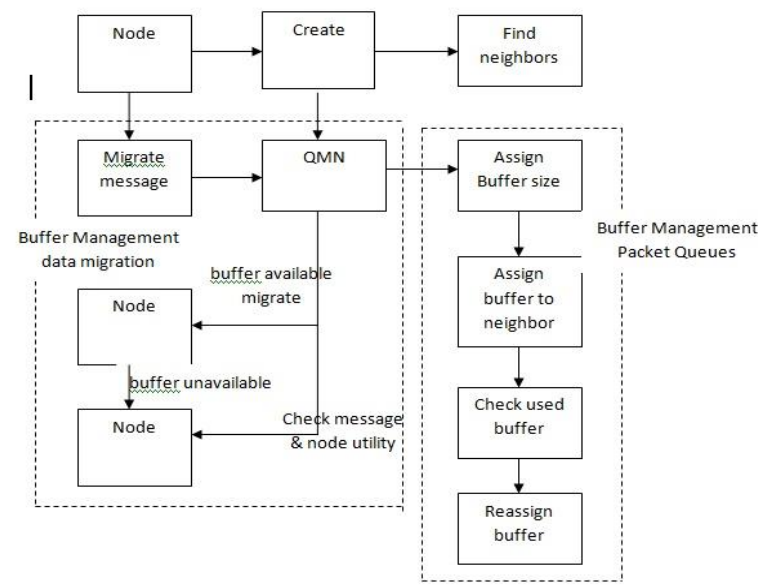

Fig. 2.System architecture of proposed Scheme

\section{PERFORMANCE EVALUATION}

The performance of projected research work is compared with existing techniques such as Drop Tail, PAQMAN and QMN. Eexperimental set up is done suing NS2 with wireless network configuration containing 50 nodes with AODV routing protocol. 50 nodes are divided as clusters with one centrally communicating node QMN (Queue management node) and few neighbor nodes. Node 0 to 9 of total 10 nodes acts as QMN nodes. Every QMN is allocated 100 buffer space of size, which equally splits for the nodes that are neighbors to QMN. For example if QMN 0 contains 4 neighbor nodes, then each neighbor gets buffer size as 25 . Sometimes, it is necessary to consider if any one of the neighbor node approaches its maximum assignment of storage buffer space area in QMN however the space for storing that's remaining within the total storage buffer as a result of reason that, one or a lot of further neighboring nodes cannot communication or causing messages to QMN. Therefore, if we have a tendency to prohibit the node to associate degree assignment of 25 packets however the space for storing remains left within the buffer, there's an opportunity for resource underutilization. So there is a provision to extend size of the each source node until it approaches to its assigned limit.

This is how we experimented the buffer management for packet queues. Similarly, the other problem we considered is data migration i.e performed through QMN from one node to other by providing collaboration between nodes. This is done by calculating utility value of a node as well as message. For utility value calculation, we assumed $\alpha+\beta+\gamma=1$, for this we considered the values of weighted factors $\alpha=0.2, \beta=0.3$ and $\gamma=0.5$ correspondingly [6]. As the data migration is considered under a QMN, we assumed average number of hops as 1 and TTL as 1 . 
Table-I: Simulation Parameters

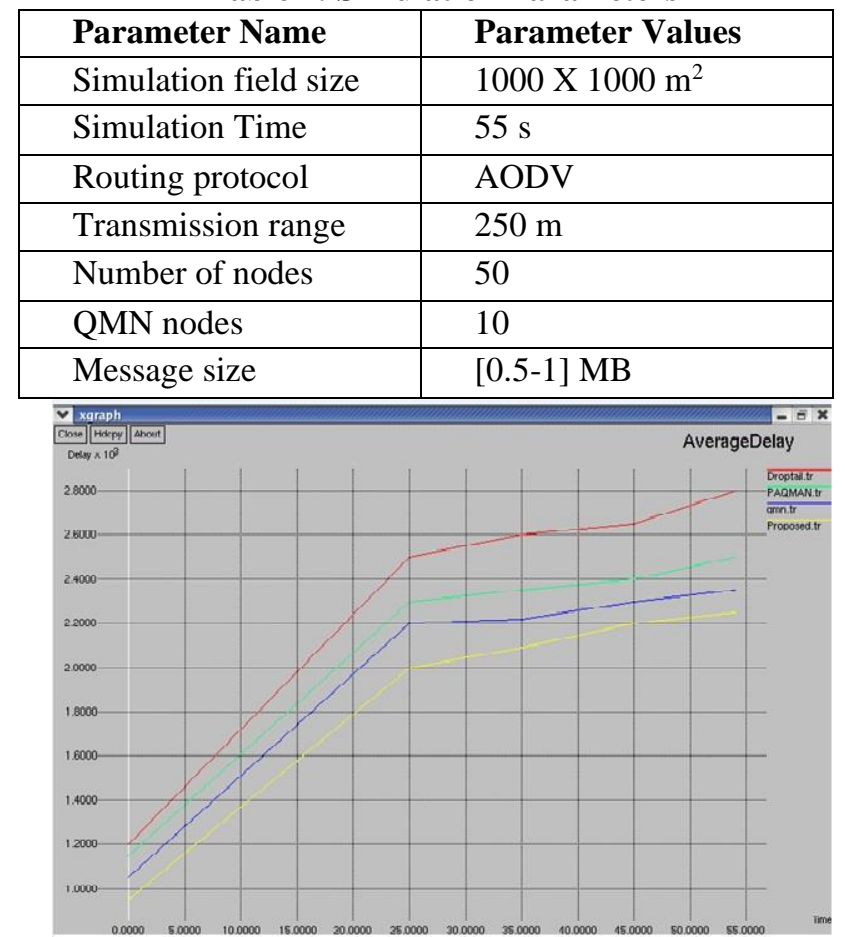

Fig. 4.Time vs End to End Delay

The result shows the Time vs End to End delay case. Simulation was carried out for 55 sec of time. Here, End to End Delay means time it takes a packet to transmit across a network from sink to destination. The proposed work exhibited less end to end delay than existing techniques.

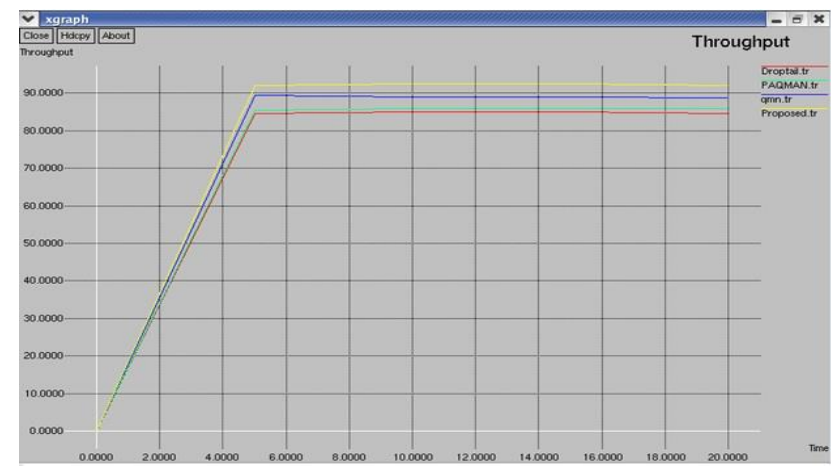

Fig.5.Time vs Throughput

The result reveals the throughput of the proposed scheme. Simulation was carried out for 55 sec of time. The amount of data transferred successfully from one place to another in a given unit of time is called throughput. The proposed work provided high throughput than existing techniques.

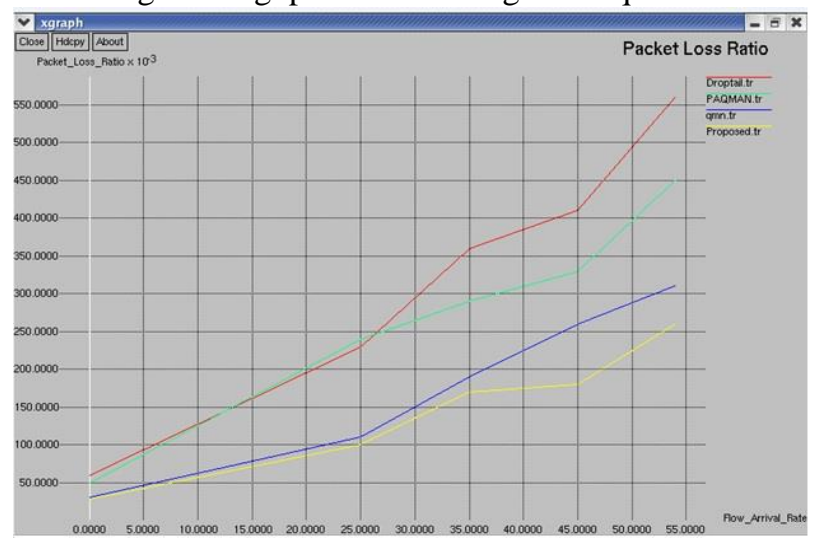

Fig. 6.Flow arrival rate vs Packet Loss
The result shows the packet loss with respect to flow arrival rate. The simulation was carried out for 55 sec of time. Packet loss is nothing but number of packets lost in network during simulation time. It is a measure of packet bits per second (bit/s). The proposed work showed low packet loss than existing techniques.

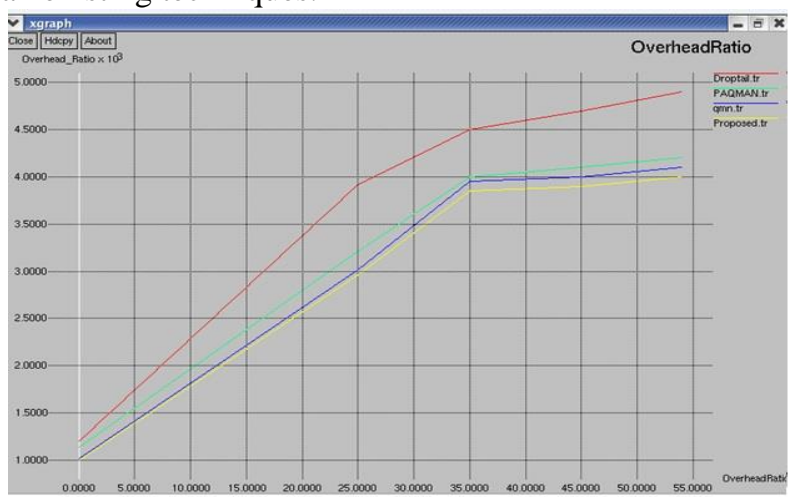

Fig. 7.Comparison of Overhead ratio

The result shows the overhead ratio of the proposed scheme. The simulation was carried out for 55 sec of time. Data that sent on each transmission contains additional information, called overhead. The proposed work incurs less overhead than existing techniques.

\section{CONCLUSION}

In this paper, we proposed a novel buffer management scheme to minimize packet loss in ad hoc wireless networks. This scheme explores the combination of QMN and CBM strategy to manage buffer size of intermediate nodes efficiently. Initially, with a centrally communicating node equal buffer storage space allocated to all its neighboring nodes. After that, the allocation is adjusted dynamically based on the prompt share of neighboring nodes and the space between utilized and assigned buffer space. By using CBM, utility value of nodes and messages calculated to forward and transfer the messages from one node to another node by providing collaboration among neighboring nodes of QMN. The simulated results show that, proposed work performance is better in terms of packet loss ratio, throughput, end to end delay and overhead ratio in a 50-node scenario compared to other existing works.

\section{REFERENCES}

1. K. D. Kumar, I. Ramya, and M. R. Masillamani, Queue management in mobile adhoc networks (Manets), in Proc. IEEE GreenCom/CPSCom 2010, 2010.

2. P. G. Kulkarni, S. I. McClean, G. P. Parr, and M. M. Black, Proactive predictive queue management for improved QoS in IP networks, in Proc. IEEE ICN/ICONS/MCL 2006, 2006.

3. R. Braden, D. Clark, J. Crowcroft, B. Davie, S. Deering, D. Estrin, S. Floyd, V. J. G. Minshall, C. Patridge, L. Peterson, K. Ramakrishnan, S.Shenker, J. Wroclawski, and L. Zhang. Rfc-2309 recommendations on queue management and congestion avoidance in the internet. Technical report, IETF, Apr. 1998.

4. S.Floyd and V.Jacobson. Random early detection gateways for congestion avoidance. IEEE/ACM Transactions on Networking, 1(4):397-413, Aug. 1993.

5. Muhammed Aamir, Mustafa A.Zaidi, “A Buffer Management Scheme for Packet Queues in MANET ," Tsinghua Science and Technology vol.18, Number: 6 , pp543-553, Dec 2013. 


\section{An Efficient Buffer Overflow Control Scheme to Mitigate Packet Loss in Manets}

6. Peiyan Yuan and Hai Yu, "A Combinational Buffer Management Scheme in Mobile Opportunistic Network" Future Internet 2017, 9, 82; doi:10.3390/fi9040082.

7. P. G. Kulkarni, M. Nazeeruddin, S. I. McClean, G. P. Parr, M. M. Black, B. W. Scotney, and P. Dini, Deploying lightweight queue management for improving performance of mobile ad-hoc networks (MANETs), in Proc. Int. Conf. On Networking and Services (ICNS), 2006.

8. J. Lutz, C. J. Colbourn, and V. R. Syrotiuk, Variable weight sequences for adaptive scheduled access in MANETs, in Sequences and Their Applications $\square$ SETA 2012, T. Helleseth, J. Jedwab, Ed. Springer Berlin Heidelberg, 2012, pp. 53-64.

9. S. Chen and B. Bensaou, Can high-speed networks survive with DropTail queues management? Comput. Netw., vol. 51, no. 7, pp. 1763-1776, 2007.

10. B. Abbasov and S. Korukoglu, Effective RED: An algorithm to improve RED's performance by reducing packet loss rate, J. Netw. Comput. Appl., vol. 32, no. 3, pp. 703-709, 2009.

11. S. Dimitriou and V. Tsaoussidis, Promoting effective service differentiation with size-oriented queue management, Comput. Netw., vol. 54, no. 18, pp. 3360-3372, 2010.

12. Krifa, A.; Baraka, C.; Spyropoulos, T. Optimal Buffer Management Policies for Delay Tolerant Networks. In Proceedings of the 5th Annual IEEE Communications Society Conference on Sensor, Mesh and Ad Hoc Communications and Networks, San Francisco, CA, USA, 16-20 June 2008; pp. 260-268.

13. Scott, K.; Burleigh, S. Bundle Protocol Specification; Internet RFC 5050. Available online: https://rfc-editor.org/rfc/rfc5050.txt (accessed on 10 November 2017).

14. Kim, D.; Park, H.; Yeom, I. Minimizing the impact of buffer overflow in DTN. In Proceedings of the $3^{\text {rd }}$ International Conference on Future Internet Technologies (CFI), Seoul, Korea, 18-20 June 2008.

15. Ayub, Q.; Rashid, S.; Zahid, M.S.M. Buffer Scheduling Policy for Opportunistic Networks. Int. J. Sci. Eng. Res.2013, 2, 1-7.

16. Rashid, S.; Ayub, Q.; Zahid, S.M.M.; Abdullah, A.H. E-DROP: An Effective Drop Buffer Management Policy for DTN Routing Protocols. Int. J. Computer. Appl. 2011, 13, 8-13.

17. Pan, D.; Ruan, Z.; Zhou, N.; Liu, X.; Song, Z. A comprehensive-integrated buffer management strategy for opportunistic networks. EURASIP J. Wirel. Commun. Netw. 2013, 2013, 103 , doi:10.1186/1687-1499-2013-103.

18. Yao, J.; Ma, C.; Yu, H.; Liu, Y.; Yuan, Q. A Utility-Based Buffer Management Policy for Improving Data Dissemination in Opportunistic Networks. China Commun. 2017, 14, 118-126.

\section{AUTHORS PROFILE}

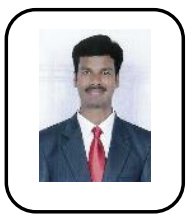

Mr. B Purushotham, received B.Tech (CSE) from J N T University, Hyderabad, India in 2005 and M.Tech (CSE) from S V University College of Engineering, Tirupati in the year 2007. He has 10 years of teaching experience. Currently he is pursuing his Ph.D in the Department of Computer Science and Engineering, S V University College of Engineering, Tirupati and also working as Assistant Professor, Department of Computer Science and Engineering, Annamacharya Institute of Technology and Sciences, Tiupati, India. His areas of interests are Advanced Computer Networks, Cloud Computing and Wireless Ad-Hoc Networks.

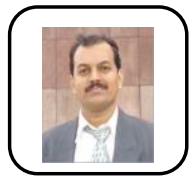

Prof. Ch D V Subba Rao has been working as a faculty member in the Dept of CSE, Sri Venkateswara University since 1992 and as Professor since 2009. His areas of interests include Distributed Systems, Peer to Peer Systems, Wireless Networks, and Advanced Computing. He published 52 papers in International/National Journals. Some of his publications appear in IEEE, ACM, Elsevier and Springer digital libraries. He guided $36 \mathrm{MTech}$ theses and $4 \mathrm{PhD}$ theses. At present he is supervising the work of $7 \mathrm{PhD}$ scholars. Prof Rao visited Austria, Hong-Kong, Netherlands, Belgium, Thailand and Germany and chaired the sessions of International Conferences viz. IEEE, IAENG and IASTED. He is serving as Member, Board of Studies in CSE/IT of Bharatiar University/Coimbatore, DU/Kuppam, RGUKT/Nuzvid, SVU/Tirupati and number of autonomous Engineering Colleges. He served as Head, Dept of CSE (for two terms), Officer-in-Charge, Computer Centre of SVUCE and Coordinator/MSIT Program. He worked as a faculty member at NIT/Warangal in 1992. His technical paper bagged best paper award in
IAENG Intl' Conference, Hong-Kong in 2008. Dr Rao is a recipient of DTA Excellence from the prestigious Delhi Telugu Academy in 2015. 